\title{
Analysing and simulating electronic devices as antennas
}

\author{
Dániel Erdősy', Tamás Bodolai², Angéla Váradiné Szarka² \\ 1 University of Miskolc, Institute of Electrical and Electronic Engineering, Miskolc-Egyetemváros 3515, Hungary \\ 2 University of Miskolc, Research Institute of Electronics and Information Technology, Miskolc-Egyetemváros 3515, Hungary
}

\begin{abstract}
Electromagnetic compatibility (EMC) is getting more and more interest with the ever-increasing adoption of electronic devices. EMC must be planned, calculated, simulated, and measured; this is necessary to ensure that the device can work in the environment of other devices and does not disturb other devices through undesired interference. In complex devices, simulation methods due to the large number of elements and complicated operations can be extremely costly and time consuming. We should consider how is it possible to reduce these costs, for example with simplifying our simulation terminology. This paper explains a general theory about using premeasured EMC properties in simulation to predict how a complex system (containing many devices) will work. This theory is about how different radiated electromagnetic waves affect other devices. EMC standards have been developed to test and limit unintentional coupling to and from electronic devices (interference), but not developed to deal with more devices crowded in a smaller environment. If we handle the different devices as antennas, in the simulation environment we must not deal with their internal structure and operation (unless we need information of their internal structure). In this way, simulation time can be drastically reduced.
\end{abstract}

\section{Section: RESEARCH PAPER}

Keywords: EMC simulation, devices as antennas, device simulation

Citation: Dániel Erdősy, Tamás Bodolai, Angéla Váradiné Szarka, Analysing and simulating electronic devices as antennas, Acta IMEKO, vol. 10, no. 3, article 21, September 2021, identifier: IMEKO-ACTA-10 (2021)-03-21

Section Editor: Lorenzo Ciani, University of Florence, Italy

Received February 8, 2021; In final form August 4, 2021; Published September 2021

Copyright: This is an open-access article distributed under the terms of the Creative Commons Attribution 3.0 License, which permits unrestricted use, distribution, and reproduction in any medium, provided the original author and source are credited.

Corresponding author: Dániel Erdősy, e-mail: elkedani@uni-miskolc.hu

\section{INTRODUCTION}

In all departments of electronic design and manufacturing, electromagnetic compatibility (EMC) is one of the most important factors. All the devices need EMC to work properly. In fact, some devices are prone to interfere with others, while others radiate a considerable amount of electromagnetic waves. Nowadays, EMC has become increasingly important, because of the growing number of electronic devices, gadgets, smart devices we use.

The basic idea is that if we know some radiation or immunity properties of the product, we could feed these into the simulation software. If we have the proper structural model of the product, then we could simulate and analyse the properties; therefore, with the results, we can consider what changes must be made. An example is when a bigger structure, which contains metal parts, magnets, external wires and electronics, may be too complicated for complex simulations, but the different parts can be analysed as simple individual antennas. This paper explains the theory behind a new method to reduce costs on electronic design stages, simulations and EMC measurements.

\section{EMC PROBLEMS IN LONG TERMS}

A common approach to achieve the required EMC levels starts with the design of the device. At this stage, most of the work that affect EMC properties can be carried out easily, without further costs. After the design, prototypes can be manufactured and, if required, EMC measurements are performed. If everything goes right, final manufacturing starts, and the product could get on the market. But what happens if after some time, we want this product to achieve higher EMC limits in special appliances, due to local laws, or other devices working next to it, [1]?

The costs of a whole new process easily get high but improving the good working product can be worth it. Here comes a special idea about this improvement: if we know what we want to improve in the product, for example, in a frequency range it emits more EM waves than we want, we could analyse the structure directly as some antennas on that specific range. EMC measurements can declare the radiation characteristic, but it could be cost effective, if we could easily simulate that. Simulation is one side of determining the radiation properties of 


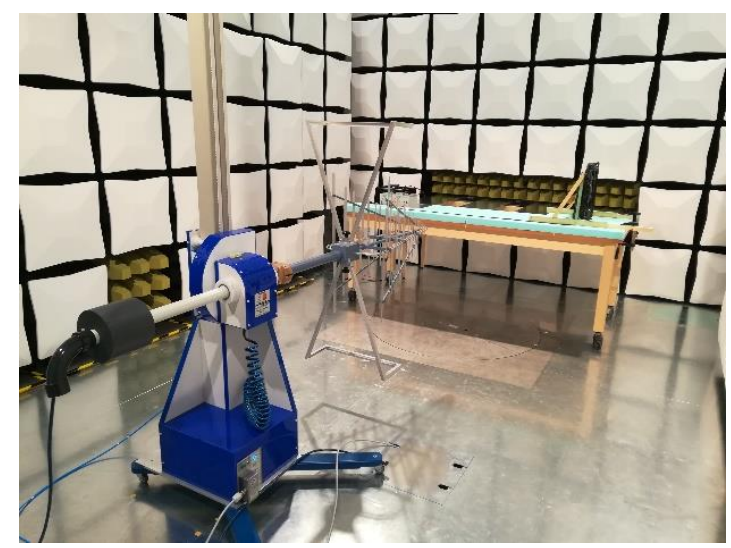

Figure 1. SAC-3 chamber with automotive table and bilog antenna at the University of Miskolc.

the product, but in function of internal structure and operation parameters, simulation time and computer requirements increase [2].

\section{DEVICES AS ANTENNAS}

Antennas can be made of a simple wire or complex metal structures, maybe some smaller blocks that act like a grid antenna. It is possible to look at our device as an antenna. They are not optimized to work as antennas (in general conditions), but as unintentional antennas.

\subsection{Gain and radiation pattern}

Antennas are typically designed for specific usage with needed characteristics (like gain and pattern). This means they have a known frequency response, directivity, or pattern. On the other hand, simple devices are not designed like this, so the mentioned parameters work on a smaller scale. This means that measurements on these unintentional antennas present some challenges compared to normal antenna tests. For example, commercial EMC tests measure the entire 3D radiation, while in automotive tests only a few directions must be measured. This is one reason why alternative methods to estimate the directivity, radiation pattern and other needed parameters are considered [3]. For real-life antennas used nowadays, well-known methods are developed to determine the required parameters and these methods (with some modifications) should be use on unintentional antennas also [4].

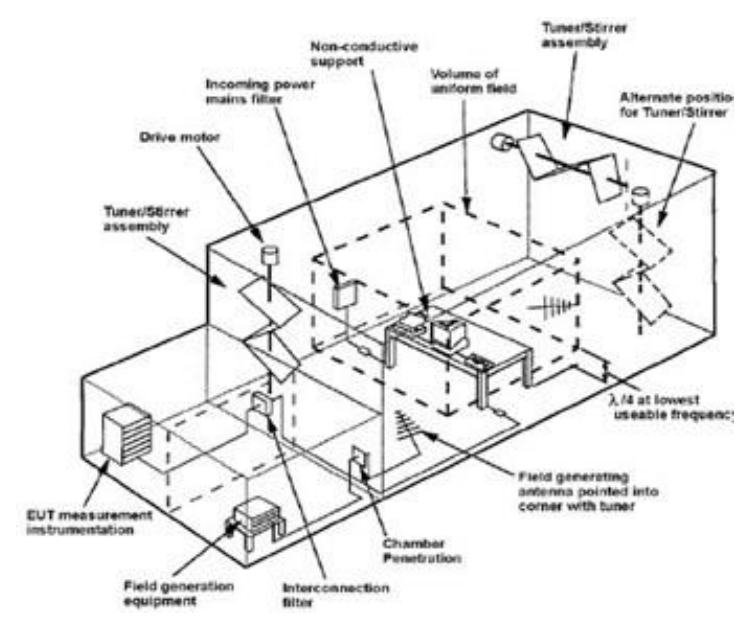

Figure 2. The reverberation chamber as described in IEC-61000-4-21 [6].

\subsection{Measuring the device}

To measure the radiated power pattern (of any device) one method is to use a fully or semi anechoic chamber (for example a SAC-3) (Figure 1) [5].

The receiving antenna must be equally far from the source. The equipment under test (EUT) is mounted on a rotating table, while the receiving antenna mounted on vertical sliding base to move up and down. Each measurement is carried out in one position, then rotating the EUT or lifting the antenna. From the measurements, a ratio of the maximum to average power can be calculated for each planar cut.

Another method uses a reverberation chamber (Figure 2). This type of chamber is generally employed for immunity testing; however, it can also provide an efficient method for radiated emission measurements. Results of these measurements yield the total radiated power, not directivity properties.

\section{SIMULATING EMC PROPERTIES}

Without EMC measuring technology, electromagnetic simulation of the device can also provide us some useful, antenna-like properties [7]. There are several types of simulating software on the market. Numerical simulations can provide full EMC properties, but to inspect a bigger complex system, it needs lots of computing time. Even though precise and complex simulation is not the most cost-effective solution, on a lower, individual parts level can be worth it. Numerical simulations are useful for both purposes, i.e. not only to represent the observed phenomena and find effective countermeasures early, but also to predict phenomena without measurement in the design stage [8].

Modelling a BLDC motor especially for EMC simulation is presented in [9]. BLDC motors are a good point in the EMC topic, because they get more and more popular, but also due to their working conditions, can suffer from lots of electromagnetic problems. The sum of instantaneous voltage of three-phase windings of a motor (also 2-phase windings), or common-mode voltage, is not zero in this system. Furthermore, the switching frequency is increasing with the development of switching devices such as IGBTs. These facts cause high-frequency leakage current, which leads to EMI/EMC issues. The common-mode voltage also provokes a shaft voltage, which causes a bearing current. The bearing current damages a bearing and makes its lifetime shorter. In addition, high-frequency switching causes overvoltage and turn-to-turn voltage stress in stator windings, which affects its insulation seriously. It is possible that modern simulation software on a fast-enough computer can simulate the most required parameters, but in [9] the entire simulation process is taken in different parts to increase efficiency. Although models are made for electromagnetic field analysis, capacitance is independently calculated by static electric field analysis and inductance with resistance is calculated by magnetic field analysis.

The motor with laminated iron core radiates different EM frequencies, because of different reasons. The effect of eddy current becomes significant only above $1 \mathrm{kHz}$. Magnetic flux is not completely pushed out of the iron core even at $1 \mathrm{MHz}$. The resistance in case of laminated iron core is larger than that in case of bulk iron core above $20 \mathrm{kHz}$. This is because the surface area where eddy current flows is larger and thus the total power loss is larger in laminated iron core for such high frequencies. In this way, with 2-D mesh data, it is likely to overestimate the shielding effect of eddy current in laminated iron core. Therefore, we should use 3-D mesh data to calculate inductance and resistance of stator windings accurately (Figure 3) [9]. This example shows 


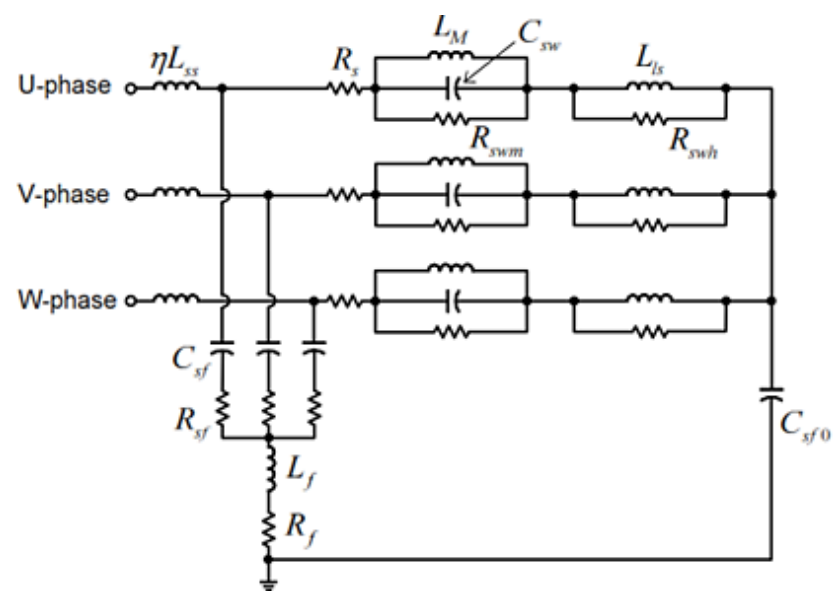

Figure 3. Equivalent circuit of a three-phase Y-connected BLDC motor [14]

that precise simulation requires different approaches; there is no straightforward solution for it. With the different simulation results it is possible to construct a high-frequency motor equivalent circuit (or model) (Figure 3 shows an example for a BLDC motor) [10].

This also means that $2 \mathrm{D}$ and $3 \mathrm{D}$ simulation software is no longer needed, because we only need the results of previous simulations. The equivalent circuit contains the data of resistance, inductance, and capacitance of the stator windings, so after some "basic" calculation we can achieve a frequency characteristic of the motor impedance (Figure 4).

Both the magnitude and the phase angle can be calculated; therefore, the whole process can result in the required antenna parameters. These numerical simulations are not relevant in fine tuning a real motor, because there are more phenomena (such as hysteresis, permeability, magnetic saturation) and lack of factors like manufacturing imperfection, temperature, aging. Nonetheless, it is possible to use the results further for our needs [11]-[13].
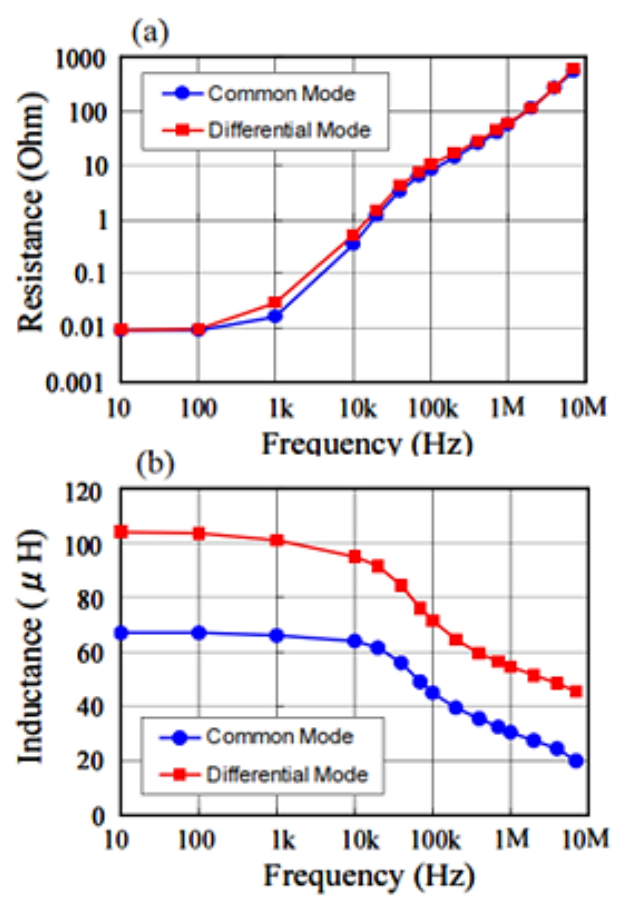

Figure 4. Calculated (a) resistance and (b) inductance of stator windings per phase for common mode and differential mode [9]

\section{ESTIMATION OF DIRECTIVITY}

After the EMC measurements or similar simulations mentioned in section 3 and 4, enough data is gained to move to the next step. This section is based on [15], [16], where a theory of estimating not all antenna parameters, but the directivity was presented (note that if directivity measurements are done, this step can be skipped.)

Directivity of an antenna (or device as antenna) is the evaluation of far-field form of the spherical mode expansion of the emitter. The assumption is the expansion coefficients are independent random variables. Equation (1) shows the general form for the far-field pattern (in 3D spherical coordinate system expressed with polar coordinates).

$$
\bar{E}(r, \theta, \vartheta)=k \sqrt{\eta} \frac{1}{\sqrt{4 \pi}} \frac{\mathrm{e}^{j k r}}{k r} \sum_{s m n} Q_{s m n}^{(3)} \overline{K_{s m n}}(\theta, \vartheta),
$$

where the spherical coordinate system is defined as usual, $\eta$ is the free space wave impedance,

$$
\sum_{s m n}=\sum_{s=1}^{2} \sum_{n=1}^{N} \sum_{m=-n}^{n}, N \approx k a,
$$

where $a$ is the radius of the minimum sphere enclosing the emitter (it is assumed the spherical coordinate system is centred in this sphere), $k a$ is called the electrical size, $Q_{s m n}^{(3)}$ are the wave coefficients, and $\overline{K_{s m n}}(\theta, \vartheta)$ are the far-field large argument, forms of the dimensionless power-normalized spherical wave functions [17]. This convention yields the expression (3)

$$
P_{\text {rad }}=\frac{1}{2} \sum_{s m n}\left|Q_{s m n}^{(3)}\right|^{2} \text {. }
$$

Expression (3) shows the total radiated power $\left(P_{\text {rad }}\right)$ from the emitter. In general, the summation over $n$ goes to infinity; however, spherical wave functions with indices $n>k a$ are cut off and will not contribute in the far-field and, because of this, the series can be truncated.

$$
N_{m}=2 \sum_{n=1}^{N}(2 n+1)=2\left(N^{2}+2 N\right),
$$

where $N_{m}$ is the total number of modes. Each coefficient has an independent real and imaginary part. With this expression, an upper bound can be found. Directivity is the ration of radiated power (at a given angle) and distance to the total radiated power divided by the total solid angle. The Caucy-Schwartz inequality yields a sum over the number of modes $\left(N_{m}\right)$, which is reduced by a factor of two. The result expression below once $(k a)$ is substituted for $\mathrm{N}$ :

$$
D_{\max } \approx\left\{\begin{array}{c}
3, k a \leq 1 \\
(k a)^{2}+2 k a, k a>1 .
\end{array}\right.
$$

The only problem here is Dmax is an overestimation of the directivity of unwanted antennas. A better estimation can be derived by assuming that the spherical-wave coefficients are independent random variables.

For unintentional emitters, the mean of co- and crosspolarized terms will be equal, so the mean value of $D$ is 1 .

$\overline{D_{\text {co }}}=\overline{D_{\text {cross }}}=1 / 2$

The field components of expression (1) is the following: 


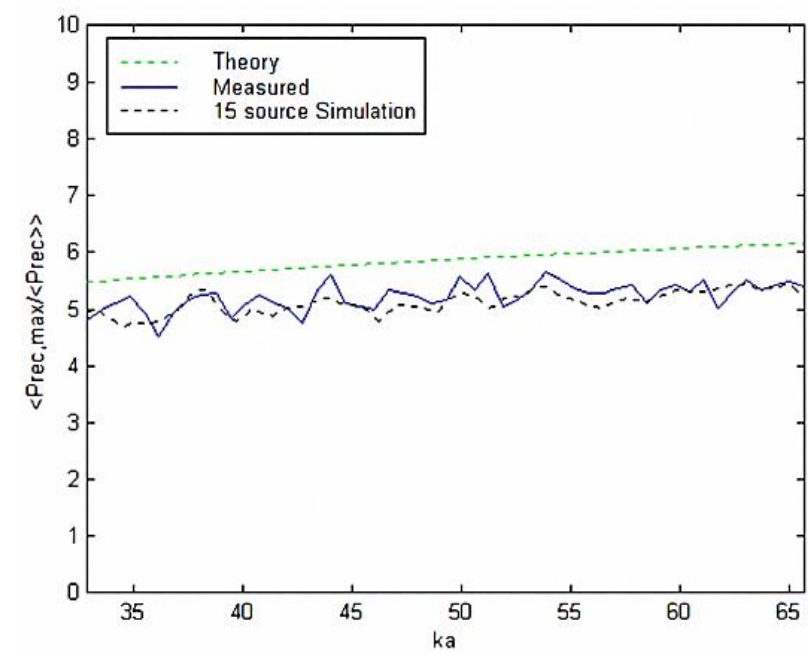

Figure 5. The estimated (theory), measured, and simulated ratio for an example EUT [3].

$$
\begin{aligned}
& \left|\overline{E_{\theta}}(r, \theta, \vartheta)\right|^{2}=\frac{\eta}{4 \pi r^{2}}\left|\sum_{s m n} Q_{s m n}^{(3)} \bar{K}_{s m n}(\theta, \vartheta) \cdot \bar{\alpha}_{\theta}\right|^{2} \\
& \left|\overline{E_{\vartheta}}(r, \theta, \vartheta)\right|^{2}=\frac{\eta}{4 \pi r^{2}}\left|\sum_{s m n} Q_{s m n}^{(3)} \bar{K}_{s m n}(\theta, \vartheta) \cdot \bar{\alpha}_{\vartheta}\right|^{2}
\end{aligned}
$$

As mentioned earlier, the real and imaginary parts of $Q_{s m n}^{(3)}$ are independent and Gaussian distributed with zero mean. The directivities $D_{\text {co }}$ and $D_{\text {cross }}$ is similarly distributed. The expected value for the maximum over $N_{s}$ samples of a $X^{2}$ with two degrees of freedom distribution is:

$$
\overline{D_{\text {co,max }}}=\overline{D_{\text {co }}} \sum_{n=1}^{N_{s}} \frac{1}{n} \text {. }
$$

This summation can be approximated and substituting the expected value of $D_{c o}$ from (6) yields expression (10).

$$
\overline{D_{\text {co, } \max }} \approx \frac{1}{2}\left[0.577+\ln \left(N_{s}\right)+\frac{1}{2 N_{s}}\right]
$$

If $k a>1, N_{s}=2 N_{m}$ and $N=k a$ expression (10) results in (4). For $k a=1$ ( $N_{s}=12$ ), (10) yields $\overline{D_{\text {co, } \max }} \approx 1.55$.

A physical interpretation of $N_{s}=12$ for $k a \leq 1$ is that the real and imaginary parts of six dipole moments (three electric and three magnetic) yield 12 independent source contributions. The directivity estimate is also very near the directivity of a single short dipole $(D=1.5)$. Thus, using 1.55 for electrically small emitters in (10) results in a continuous function and should give good estimates for directivity:

$$
\begin{aligned}
& \overline{D_{\max }} 1.55, k a \leq 1 \\
& \approx\left\{\frac{1}{2}\left[0.577+\ln \left(4(k a)^{2}+8 k a\right)+\frac{1}{8(k a)^{2}+16 k a}\right], k a>1 .\right.
\end{aligned}
$$

The main difference between the intentional emitter upper bound given by (5) and the unintentional emitter expected value given by (11) is that the upper bound increases rapidly as the square of the electrical size $k a$, while the expected value increases only as the natural logarithm (ln) of electrical size $k a$.
The estimated, measured and simulated total radiated power ratio for an example EUT are compared in [3].

Figure 5 shows that the measurement result and simulation result are in optimal agreement; also, the theoretical estimation is adequately good. This means that estimation can be used instead of measurement and simulation, but if higher precision is needed, they cannot be ignored.

The theory also shows that, in earlier stages, only estimation can be enough to determine the overall EMC performance. This can further reduce costs, because we do not need actual measurements or time-consuming simulation processes to get antenna like properties.

On the other hand, a large and complex device containing many different parts is usually made of such individual components that their EMC properties are measured by the manufacturer (for example, in automotive industry, every electronic component should pass EMC restrictions before building into the actual vehicle), so this type of estimation or simulation process can be skipped [18].

\section{ANTENNA ARRAYS}

At his point, we should have all the parameters to handle our individual devices as antennas. Measurements, simulations, and estimations must give enough information about the product. When assembling the final, complex device made of the individual devices we can treat it like an antenna array. A classical antenna array (often called a 'phased array') is a set of two or more antennas. The signals from the antennas are combined or processed to achieve improved performance over that of a single antenna. These types of antennas can be used to increase overall gain, maximize the signal to interference plus noise ratio, direct the array so that it is most sensitive in a direction, cancel out interference and so on [19]. These applications (some of them with reciprocity) are also the basics of decreasing overall EMC performance of a complex system. In a phased array it is not so hard to add together the different antennas, because they are usually consisting of similar ones and made symmetrically [4], [20].

Classical antenna arrays are made for specific reasons, but in our case, the different devices according to their operation, orientation, and distance from each other act like transmitters, but also receivers [21]. If we choose one operation where we handle all of them as receivers, we can calculate the EMI properties. Next to the weighting, we need to include distance and directivity factors to get accurate results. We can calculate the overall power of radiated electromagnetic waves if we handle all of them like transmitters. And finally, if we want a mixed operation calculation, because we know that some of the individual devices work more like transmitters (emits more EM waves than they are immune to) and some of them work more like receivers (they are sensitive to EM waves but does not radiate so much) we need calculate them one by one. This method is like superposition in electrotechnics but with antennas [18].

Weighting, antenna factors, distances, directivity properties must stay, but they can be "switched off" one-by-one and in a round calculate the effect on all the others, then finally sum-up the results of each round. In each iteration, it is possible to inspect the effect of only one device on the others or choosing several iterations to select a group of devices. It yields a similar result if we take the complex system; put it in an EMC chamber and measuring. After one complete measurement, we switch off some devices, or just leave a little group of devices and measuring 
again the EMC. The cost of only calculations and measurements cannot be compared [22].

\section{POSSIBLE ERRORS}

This general theory is based on devices as antennas so one thing must be mentioned is conducted EMC [23]. It is acceptable to measure the connecting wires, but real-life application may use cables different in length, diameter, shielding properties, etc. Although we know the radiation pattern and radiated immunity of the product, but if we want proper results of the simulation, we must inspect the connecting wires also. It is not the most important in all applications but for example in automotive industry where lots of wires inside a metal body crowded with electronics, the problem should be solved [24], [25].

Another possible factor is the passive, shielding like parts in the complex system. Mountings, covers, design elements made from metal can easily distort the results. If we take them in count like the "active" devices, the risk of error decreases, but this procedure highly enlarges the number of devices.

The less precise properties we use, the less precise results we get. If EMC measurements or proper simulations are not available, we can estimate the performance of the device but in the more cases we calculate with estimations, the results may not meet our expectations in precision.

\section{CONCLUSIONS AND OUTLOOK}

This paper provided a theory of handling devices as antennas to easily calculate EMC properties if more devices are used in a closer area. The individual steps are used commonly, but until this point, we have not found this combined procedure.

The scenario appears easily in electrical boxes, vehicles or in our house because we use more and more electronic devices. To achieve results, we need antenna like properties of the device, like overall gain, frequency chart, directivity, and other factors. These can be gained from measurements in proper EMC chamber, 3D simulations and calculations or estimations. With these we must calculate the final performance of this antenna array with different methods depend on what performance

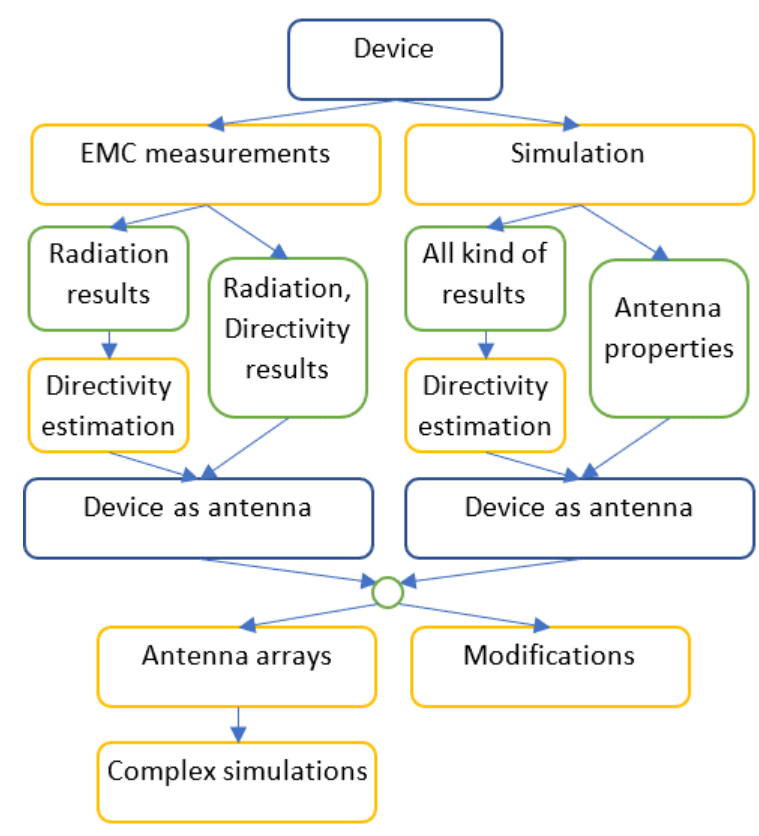

Figure 6. An illustration showing the whole concept. parameter we need. The whole concept is illustrated in Figure 6. Further research on this theory must be done because the whole process (in ideal circumstances) can easily cut-off costs in designing and EMC testing. Also, to prove the concept example systems should be build up to try the different approaches.

\section{REFERENCES}

[1] R. G: Jobava, A. L. Gheonjian, J. Hippeli, G. Chiqovani, D. D. Karkashadze, F. G. Bogdanov, B. Khvitia, A. G. Bzhalava, Simulation of low-frequency magnetic fields in automotive EMC problems, IEEE Transactions on Electromagnetic Compatibility 56(6) (2014) pp. 1420-1430.

DOI: 10.1109/TEMC.2014.2325134

[2] A. Barchanski, EMC Simulation of Consumer Electronic Devices, High Frequency Electronics, July 2013.

[3] P. F. Wilson, D. A. Hill, C. L. Holloway, On determining the maximum emissions from electrically large sources, IEEE Trans. Electromagn. Compat. 44(1) (2002), pp. 79-86. DOI: $\underline{10.1109 / 15.990713}$

[4] C. A. Balanis, Antenna theory, Analysis and design. John Wiley \& Sons, INC., 1982 ISBN: 9780471606390.

[5] J. Glimm, K. Münter, R. Pape, M. Spitzer, P. B. Ptb, D.Braunschweig, New results of antenna calibration in a singleantenna set-up, XVI IMEKO World Congress Measurement, Vienna, Austria, 25-28 Sept. 2000, pp. 1-3. Online [Accessed 17 August 2021]

https://www.imeko.org/publications/wc-2000/IMEKO-WC2000-UNC-P507.pdf

[6] F. Leferink, High intensity electromagnetic field generation using a transportable reverberation chamber, URSI Gen. Assem. Int. Symp., Ghent, Belgium, 09-16 Aug. 2008. Online[Accessed 17 August 2021] https://www.ursi.org/proceedings/procGA08/papers/E01p6.p df

[7] M. Borsero, A. D. Chiara, C. Pravato, A. Sona, M. Stellini, A. Zuccato, Considerations about radiated emission tests in anechoic chambers that do not fulfil the NSA requirements, Proc. 16th IMEKO TC4 Int. Symp. Explor. New Front. Instrum. Methods Electr. Electron. Meas. 13th TC21 Int. Work. ADC Model. Test. - Jt. Sess. Proc., Florence, Italy, 22-24 Sept. 2008, pp. 825-830. Online [Accessed 3 September 2021]

https://www.imeko.org/publications/tc4-2008/IMEKO-TC42008-160.pdf

[8] F. G. Awan, N. M. Sheikh, J. Gohar, Simulation of a parametric model for interference cancellation in open space EMC measurement, Proc. 2010 Seventh International Conference on Information Technology: New Generations, Las Vegas, NV, USA, 12-14 April 2010, pp. 924-928.

DOI: $10.1109 /$ ITNG.2010.197

[9] K. Maki, H. Funato, L. Shao, Motor modeling for EMC simulation by 3-D electromagnetic field analysis, Proc. 2009 IEEE Int. Electr. Mach. Drives Conf. IEMDC '09, Miami, FL, USA, 3-6 May 2009, pp. 103-108. DOI: $10.1109 /$ IEMDC.2009.5075190

[10] Padmaraja Yedamale, Brushless DC (BLDC) Motor Fundamentals, Microchip. p. 20, 2004.

[11] A. Cataldo, G. Monti, E. De Benedetto, G. Cannazza, L. Tarricone, L. Catarinucci, On the use of a reliable low-cost set-up for characterization measurements of antennas, Proc. 16th IMEKO TC4 Int. Symp. Explor. New Front. Instrum. Methods Electr. Electron. Meas. 13th TC21 Int. Work. ADC Model. Test. - Jt. Sess. Proc., Florence, Italy, 22-24 Sept. 2008, pp. 62-65. Online [Accessed 3 September 2021] https://www.imeko.org/publications/tc4-2008/IMEKO-TC42008-069.pdf

[12] D. Giordano, L. Zilberti, M. Borsero, R. Forastiere, W. Wang, Validation of numerical methods for electromagnetic dosimetry through near-field measurements, ACTA IMEKO 4(1) (2016), 
pp.90-96.

DOI: $\underline{10.21014 / \text { acta imeko.v4i1.169 }}$

[13] D. Giordano, L. Zilberti, M. Borsero, R. Forastiere, W. Wang, Experimental set-up for the validation of numerical methods in electromagnetic dosimetry, 19th IMEKO TC4 Symp. - Meas. Electr. Quant. 2013 17th Int. Work. ADC DAC Model. Test., Barcelona, Spain, 18-19 July 2013, pp. 549-554. Online [Accessed 17 August 2021]

https://www.imeko.org/publications/tc4-2013/IMEKO-TC42013-130.pdf

[14] M. Gries, B. Mirafzal, Permanent magnet motor-drive frequency response characterization for transient phenomena and conducted EMI analysis, Proc. 2008 Twenty-Third Annual IEEE Applied Power Electronics Conference and Exposition, Austin, TX, USA, 24-28 Feb. 2008, pp. 1767-1775.

DOI: $10.1109 /$ APEC.2008.4522966

[15] P. F. Wilson, Radiation patterns of unintentional antennas: Estimates, simulations, and measurements, in Proc. 2010 AsiaPacific Symp. Electromagn. Compat. APEMC 2010, Beijing, China, 12-16 April 2010, pp. 985-989. DOI: $10.1109 /$ APEMC.2010.5475702

[16] G. Koepke, D. Hill, J. Ladbury, Directivity of the test device in EMC measurements, IEEE Int. Symp. Electromagn. Compat., 2 (2000), pp. 535-540. DOI: $10.1109 /$ ISEMC.2000.874677

[17] J. E. Hansen (Ed.), Spherical near-field antenna measurements, Herts, United Kingdom, The Institution of Engineering and Technology, 2008. ISBN: 086341110X DOI: $10.1049 /$ PBEW026E

[18] B. A. Austin, A. P. C. Fourie, Characteristics of the wire biconical antenna used for EMC measurements, IEEE Trans. Electromagn. Compat. 33(3) (1991), pp. 179-187. DOI: $10.1109 / 15.85131$

[19] Antenna-Theory.com, Antenna Arrays (Phased Arrays). Online [Accessed 17 August 2021] http://www.antenna-theory.com
[20] X. Wang, Z. Peng, K.-H. Lim, J.-F. Lee, Multisolver domain decomposition method for modeling EMC effects of multiple antennas on a large air platform, IEEE Trans. Electromagn. Compat. 54(2) (2012), pp. 375-388. DOI: $10.1109 /$ TEMC.2011.2161871

[21] M. T. Ma, B. K. Singaraju, Theory and applications of antenna arrays, New York, John Wiley \& Sons Inc., 1975, ISBN: 9780471557951.

[22] S. Frei, R. G. Jobava, D. Topchishvili, Complex approaches for the calculation of EMC problems of large systems, Proc. 2004 International Symposium on Electromagnetic Compatibility, Silicon Valley, CA, USA, 9-13 Aug. 2004, pp. 826-831. DOI: $10.1109 /$ ISEMC.2004.1349929

[23] A. M. Silaghi, A. P. Buta, M. Silviu Baderca, A. De Sabata, Methods for reducing conducted emissions levels, Proc. 22nd IMEKO TC4 Int. Symp. 20th Int. Work. ADC Model. Test. 2017 Support. World Dev. Through Electr. Electron. Meas., Iasi, Romania, 14-15 Sept. 2017, pp. 352-355. Online [Accessed 17 August 2021] https://www.imeko.org/publications/tc4-2017/IMEKO-TC42017-069.pdf

[24] G. Chavka, M. Sadowski, N. Litwinczuk, M. Garbaruk, Structure and EMC simulation of vehicle radiocommunication base station, Proc. IEEE 6th International Symposium on Electromagnetic Compatibility and Electromagnetic Ecology, St. Petersburg, Russia, 21-24 June 2005, pp. 111-115. DOI: $10.1109 /$ EMCECO.2005.1513077

[25] D. Senic,A. Sarolic, Simulation of a shipboard VHF antenna radiation pattern using a complete sailboat model, Proc. SoftCOM 2009 - 17th International Conference on Software, Telecommunications Computer Networks, Hvar, Croatia, 24-26 Sept. 2009, pp. 65-69. Online [Accessed 17 August 2021] https://ieeexplore.ieee.org/stamp/stamp.jsp?tp=\&arnumber $=53$ $\underline{06818}$ 